\title{
The energy levels of habitual long and short sleepers
}

\author{
ROBERT A. HICKS and MICHAEL GUISTA \\ San Jose State University, San Jose, California 95192
}

\begin{abstract}
Bihourly self-reports of alertness or energy level were obtained from groups of habitual long and short sleepers over a period of several weeks. The data support the seeming paradox that habitually less sleep results in higher levels of wakeful energy.
\end{abstract}

On the basis of clinical observations, Hartmann (1973) described a group of habitual short sleepers as "efficient, energetic, ambitious persons who tended to work hard and keep busy" (p. 62). This observation is not a unique one. For example, Terman and Hocking (1913) noted that energy levels of elementary school children seemed to be inversely related to their habitual sleep durations. Further, there are several case reports of habitual short sleepers (e.g., Dement, 1974; Jones \& Oswald, 1968) that make note of the relatively high levels of drive that these individuals seemed to bring to their wakeful activities. While observations such as these are noteworthy, there has, to our knowledge, been no objective evidence for the rather provocative hypothesis that habitual short sleep is associated with heightened levels of wakeful drive, and thus, this is the purpose of this study.

Vogel (1979) has proposed a hypothesis that is relevant to the issues under consideration here. He has argued that certain REM sleep (RS) deprivation (RSD) effects can be explained by his theory that amount of RS may play an important role in determining wakeful drive level. Specifically, he has stated that "prevention of periodic disinhibition and neural discharge during RSD increases the waking excitability of a drive's neural substrate and thereby increases the waking drive. Inversely, the theory holds that disinhibition and neural discharge during RS reduce the waking excitability of the drive's neural substrate and thereby reduce the waking drive" (Vogel, 1979, p. 235). Further, in explicating his theory, Vogel added that "the inhibitory mechanism postpones discharge of some augmented excitability to a later time. Augmented excitability is then gradually discharged as its correlated augmented inhibition gradually diminishes. In short, RSD increases waking neural excitability by interfering with its rapid discharge during waking" (p. 235). There is reason to argue, as the following paragraph suggests, that Vogel's theory can be used as a basis for predictions concerning the relationship(s) between habitual sleep duration and level of wakeful drive.

Reprint requests should be addressed to Robert A. Hicks, Department of Psychology, San Jose State University, San Jose, California 95192.
It is a well documented fact that the stages of sleep are not distributed uniformly over the normal 8-h sleep period. Most slow-wave sleep (SWS) occurs during the first one-third of this period and most RS occurs during the last one-third of this period. Thus, if sleep is shortened, the amount of SWS remains relatively constant, but the amount of RS is reduced in proportion to the reduction in total sleep time. This relationship can be clearly demonstrated if one examines the mean amounts of these stages of sleep, derived by compiling data from four studies (i.e., Hartmann, 1973; Jones \& Oswald, 1968; Stuss \& Broughton, 1978; Webb \& Agnew, 1970) in which polygraphic recordings were obtained of the sleep of habitual long and/or short sleepers. Overall, the mean amounts of SWS that we computed from these studies for their long and short sleepers were $82 \mathrm{~min}$ and $83 \mathrm{~min}$, respectively. However, the amounts of RS for these long and short sleepers were $138 \mathrm{~min}$ and $56 \mathrm{~min}$, respectively. Based on the collective results of these studies, it seems warranted to argue that a primary difference in the sleep of habitual long and short sleepers is their nightly amounts of RS. Thus, using Vogel's (1979) theory, we predicted that habitual short sleepers would report higher levels of energy than habitual long sleepers over their entire period of wakeful activity.

\section{METHOD}

\section{Subjects}

Our subjects were seven habitual long sleepers (median sleep duration $\geqslant 8.5 \mathrm{~h} /$ night) and nine habitual short sleepers (median sleep duration $\leqslant 6.5 \mathrm{~h} /$ night). These subjects were selected from a much larger group of university students on the basis of their responses to a sleep habits questionnaire (see Hicks, Allen, Armogida, Gilliland, \& Pellegrini, 1980). Selected for further study were those students who met either of the aforementioned habitual sleep duration criteria, were satisfied with the length and quality of their sleep, had well established sleep habits (i.e., had experienced their current sleep duration for at least a 6-month period), and were willing to volunteer for the demanding daily record keeping required by the design of this study for at least a 30 -day period.

\section{Materials and Procedure}

Subjects were asked to keep a daily sleep diary. In their diaries, they recorded total sleep time and any unusual events 
Table 1

Mean Alertness Levels, Standard Deviations, and ts Between Means for the Long and Short Sleeper for Each Hours Since Arising Interval

\begin{tabular}{|c|c|c|c|c|c|c|c|c|c|c|c|c|c|c|}
\hline \multirow[b]{3}{*}{ Sleep Group } & \multicolumn{14}{|c|}{ Hours Since Arising Interval } \\
\hline & \multicolumn{2}{|c|}{$1-2$} & \multicolumn{2}{|c|}{34} & \multicolumn{2}{|c|}{$5-6$} & \multicolumn{2}{|c|}{$7-8$} & \multicolumn{2}{|c|}{$9-10$} & \multicolumn{2}{|c|}{$11-12$} & \multicolumn{2}{|c|}{$13-14$} \\
\hline & Mean & SD & Mean & SD & Mean & SD & Mean & SD & Mean & SD & Mean & SD & Mean & SD \\
\hline Long Sleepers & 2.4 & 1.3 & 1.9 & 1.1 & 1.8 & 1.2 & 1.9 & 1.2 & 1.9 & 1.2 & 2.3 & 1.5 & 2.8 & 1.4 \\
\hline Short Sleepers & 2.4 & 1.3 & 1.7 & .9 & 1.7 & .9 & 1.9 & 1.0 & 2.1 & 1.1 & 2.0 & 1.2 & 2.1 & 1.2 \\
\hline Long-Short t & .17 & & $2.90 \dagger$ & & 1.60 & & .18 & & 1.18 & & $2.17 *$ & & $5.46 \dagger$ & \\
\hline
\end{tabular}

${ }^{*} p<.05 . \quad t p<.01$.

that could distort normal sleep duration (e.g., prolonged strenuous exercise, the use of certain drugs, and excessive stress or depression). Subjects were also asked to respond to the Stanford Sleepiness Scale (SSS; Hoddes, Zarcone, Smythe, Phillips, \& Dement, 1973) every other hour during the entire daily period of wakefulness.

The SSS is a 7-point Likert-type scale that has been used in numerous studies as a measure of sleepiness, or conversely, as is the case here, as a measure of alertness. In responding to the SSS, subjects rated their perceived alertness from a score of 1 (i.e., "feeling active and vital; wide awake") to a score of 7 (i.e., "almost in a reverie, sleep onset soon; lost struggle to remain awake"). We exclude from consideration those responses to the SSS that were made subsequent to the experience of any unusual sleep-distorting event. In all, the long sleepers contributed 244 subject-days of data, and 285 subject-days of data were collected from the short sleepers.

\section{RESULTS AND DISCUSSION}

The SSS data were organized into 2 -h blocks that started at the time of arising. These data are summarized in Table 1. Please note that when we defined the range of 2 -h intervals that were used to form Table 1 , we selected only those intervals for which there were a substantial number of responses from both the longand short-sleeper groups.

The data summarized in Table 1 were analyzed by computing a 2 (sleep duration) by 7 (hours since arising) factor analysis of variance. The sleep duration and hours since arising main effects were both significant, as was the Sleep Duration by Hours Since Arising interaction $[F(1,3052)=14.07, \quad p<.001 ; \quad F(6,3052)=24.67$, $\mathrm{p}<.001 ;$ and $\mathrm{F}(6,3052)=5.46, \mathrm{p}<.01$, respectively] .

The significant main effect for sleep duration resulted from the fact that overall, the short sleepers rate themselves as more alert and energetic than the long sleepers rate themselves. This in spite of the fact that the short sleepers average at least $2 \mathrm{~h}$ less sleep than the long sleepers. This finding is consistent with the predictions made from Vogel's (1979) hypothesis.

The significant main effect for hours since arising was anticipated and is irrelevant to the hypothesis of this study. This result simply reflects the well established fact that level of alertness fluctuates systematically over the daily period of wakefulness.

The significant interaction between habitual sleep duration and hours since arising was not anticipated. To elaborate the reasons for this interaction, we com- puted separate $t$ statistics between the alertness means of the long and short sleepers for each hours-since-arising interval. These values are listed in Table 1. By inspecting Table 1, one can see that there were two distinct periods (i.e., 3-6 and $11-14 \mathrm{~h}$ since arising) during which the short sleepers were clearly more alert and energetic than the long sleepers. During the remaining intervals, these groups were about equal in self-reported alertness. Thus, the pattern of the means that contributed to this significant interaction is not entirely consistent with the predicted sustained advantage in energy level for the short sleepers. However, in this regard, it should be noted that the greatest differences in alertness between the long and short sleepers occurred toward the end of the period of wakefulness. Therefore, it seems fair to conclude that our data provide reasonably good support for both of the inferences drawn from Vogel's (1979) theory and offer further evidence in support of the seeming paradox that habitually shortened sleep may lead to greater levels of energy.

\section{REFERENCES}

Dement, W. C. Some must watch while some must sleep. San Francisco: Freeman, 1974.

Hartmann, E. The functions of sleep. New Haven, Conn: Yale University Press, 1973.

Hicks, R. A., Allen, J. G., Armogida, R. E., Gilliland, M. A., \& Pellegrini, R. J. Reduction in sleep duration and Type A behavior. Bulletin of the Psychonomic Society, 1980, 16, 109-110.

Hoddes, E., Zarcone, V., Smythe, H., Phillips, R., \& Dement, W. C. Quantification of sleepiness: A new approach. Psychophysiology, 1973, 10, 431-436.

Jones, H. S., \& Oswald, I. Two cases of healthy insomnia. Electroencephalography and Clinical Neurophysiology, 1968, 24, 378-380.

Stuss, D., \& Broughton, R. Extreme short sleep: Personality profiles and a case study of sleep requirement. Waking and Sleeping, 1978, 2, 101-105.

Terman, L. N., \& Hocking, A. The sleep of school children: Its distribution according to age, and its relation to physical and mental efficiency. Journal of Educational Psychology, 1913, 4, 138-147.

Vogel, G. W. A motivational function of REM sleep. In R. Drucker-Colin, M. Shkurovich, \& M. B. Sterman (Eds.), The functions of sleep. New York: Academic Press, 1979.

Webb, W. B., \& Agnew, H. W. Sleep stage characteristics of long and short sleepers. Science, 1970, 168, 146-147.

(Received for publication January 11, 1982.) 